\title{
Study On The Evaluation Of Supply Chain Partner Selection
}

\author{
Yewang Zhou \\ School of Business, Huanggang Normal University, Huanggang city, 438000,china \\ Email:whuw@sina.com
}

\begin{abstract}
With the aggravation of global competition and progress of the science and technology, modern management thought and means are improving and developing constantly, more and more enterprise begin to use supply chain management tactics. Partner selection play a very important role in supply chain management, it determine whole competitive power of supply chain directly, so, selecting a suitable method of partner selection become an important component of the modern enterprise strategy. This paper has introduced two-phase method when selecting partner. the first phase classify all partners use grey clustering and select strategic partner ,on the basis of this ,the second phase determine the optimal partner and quantity of each partner use model of double-layer programing, then use genetic algorithm solve the model, in the last the paper set a example to demonstrate validity of the method.
\end{abstract}

Keyword-supply chain, partner selection, grey clustering; double-layer progrming, genetic algorithm.

\section{INTRODUCTION}

Supply chain is a kind of network structure formed in the course of production and circulation, it includes main participants such as raw materials supplier, accessory supplier, scientific research institution, manufacturer, seller, customer, etc.. Supply chain cooperation partnership relation is a long-term strategy relations built in the same supply chain between upper layer and lower layer. Establishing good supply chain partnership will help to reduce cost, shorten reaction time and create new market value etc., the key question of supply chain management is how to choose the ideal cooperative partner, if you want to make supply chain have strong competitiveness, enterprise in the supply chain should form good partnership relation, only good partnership be established, could make supply chain work effectively ${ }^{[1]}$.

Whether could succeed in establishing partnership relation in supply chain, its key lies in the selection of cooperative partner ${ }^{[2]}$. How to select cooperative partner have been already studied in before relevant literature, but research in the past was mostly to calculate the best cooperative partner as the final choice through a concrete method, but actually in practical application, this method has much limitation. The paper put forward two-phase method, the first phase is to classify all partner and select the good partners as the strategic cooperative partner, then accordingly to different strategies, determine final partner and quantity of every partner.

\section{THE FIRST PHASE SELECTION BASED ON GREY CLUSTERING}

Our important task. is that confirm strategic cooperative partner in the initial stage of establishment of supply chain. Enterprises select partner is a complicated dynamic multifactor comprehensive decision question, the message that the policymaker receives is incomplete, is grey information ${ }^{[3]}$. So applying grey systematic theory for selecting partner and solving the problem is a suitable method.

\section{A Set Up Coefficient Matrix}

Suppose there is s clustering target, i.e. s projects of partner selection ; Each project has m clustering index; $\mathrm{N}$ different grey clustering, $d_{i j}$ is whighing weighed value for NO.i clustering target to NO.j clustering index $i=1,2,3, \cdots s, j=1,2,3, m$,D is the matrix which is made up of the element $d_{i j}$, is called coefficient matrix:

$$
D=\left[\begin{array}{cccc}
d_{11} & d_{12} & \cdots & d_{1 m} \\
d_{21} & d_{22} & \cdots & d_{2 m} \\
\cdots & \cdots & \cdots & \cdots \\
d_{s 1} & d_{s 2} & \cdots & d_{s m}
\end{array}\right]
$$

B Determine Grey Whitening Weighed Function Of Each Index $f_{i j}$

Generally speaking, whitening weighed function is divided into three kind ,its function image is as followed:

Figure 1 Three kind whitening weighed function
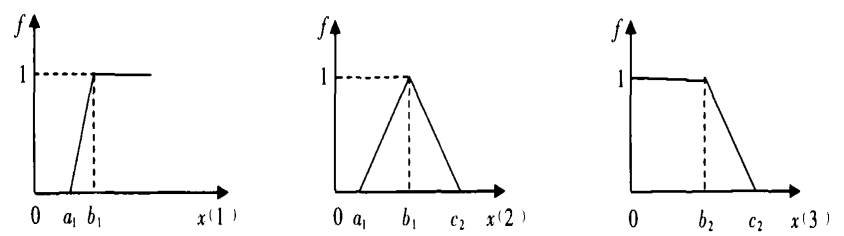

It is the described separately above three kinds writening weighed function

$$
f^{1}:\left[a_{1}, b_{1}, \infty\right], f^{2}:\left[a_{1}, b_{1}, c_{2}\right], f^{3}:\left[0, b_{2}, c_{2}\right]
$$




$$
\begin{gathered}
f^{1}(x)=\left\{\begin{array}{cc}
0, & x<a \\
x-a_{1} / b_{1}-a_{1}, & x \in\left[a_{1}, b_{1}\right] \\
1, & a \geq b_{1}
\end{array}\right. \\
f^{2}(x)=\left\{\begin{array}{cc}
0, & x \notin\left[a_{1}, c_{2}\right] \\
x-a_{1} / b_{1}-a_{1} & x \in\left[a_{1}, b_{1}\right] \\
c 2-x / c_{2}-b_{1} & x \in\left[b_{1}, c_{2}\right]
\end{array}\right. \\
f^{3}(x)=\left\{\begin{array}{cc}
0 & x \notin\left[0, c_{2}\right] \\
1 & x \in\left[0, b_{2}\right] \\
c_{2}-x / c_{2}-b_{2} & x \in\left[b_{1}, c_{2}\right]
\end{array}\right.
\end{gathered}
$$

\section{Calculate Clusteing Weighed Matrix}

$$
\omega_{j k}=\lambda_{j k} / \sum_{j=1}^{m} \lambda_{j k}, \omega_{j k} \text { is known as NO. grey }
$$

clustering weigh of $\mathrm{j}$ index, $\lambda_{j k}$ is NO. $\mathrm{k}$ grey clustering critical value of $\mathrm{j}$ index. in this equatation

$$
\lambda_{j k}=\left\{\begin{array}{cc}
1 / 2\left(b_{1}+b_{2}\right), & f_{j k}=f^{0}:\left[a_{1}, b_{1}, b_{2}, c_{2}\right] \\
b_{1}, & f_{j k}=f^{1}:\left[a_{1}, b_{1}, \infty\right] \\
b_{1}, & f_{j k}=f^{2}:\left[a_{1}, b_{1}, c_{2}\right] \\
b_{1}, & f_{j k}=f^{3}:\left[0, b_{2}, c_{2}\right]
\end{array}\right.
$$

D Ask Clustering Coefficient And Carry On Clustering

$$
\sigma_{i k}=\sum_{j=1}^{m} f_{j k}\left(d_{i j}\right) \omega_{j k}, \omega_{j k} \text { is clustering coefficient }
$$

that NO.i clustering target correspond to NO. k grey clustering.

If there is $\sigma^{*}{ }^{i k}$ satisfy:

$$
\sigma_{i k}^{*}=\max \sigma_{i k}=\max \left\{\sigma_{i 1}, \sigma_{i 2}, \cdots, \sigma_{i k}\right\}
$$

thus clustering target i belong to grey clustering $k^{*}$.

\section{THE SECOND PHASE BASED ON DOUBLE LAYER PROGRMING}

Now strategic cooperative partner have been determined by grey clustering ,in real operation, enterprises will select several cooperative partners at the same time ${ }^{[4]}$,this will disperse risk and don't be in the control of partner. so how to distribute demand among the strategic partner is a key question. settlement will lower costs, ensure the quality of products, while can arouse the enthusiasm of cooperative partner, promote the competition between them, improve service level constantly .In this paragraph we will study selection and distribution by double-layer programing. The upper layer programing be able to describe as the decision department select the best partner to make the total cost minimum, the lower layer programing is how to distribute demand quantity among each partner. The upper layer programing model is followed ${ }^{[6]}$.

$$
\begin{aligned}
& \min F=\sum_{i=1}^{m} \sum_{j=1}^{n} c_{i j}\left(x_{i j}\right) x_{i j}+\sum_{j=1}^{n} g_{j} Y_{j}+\sum_{j}\left(t_{j} \sum_{i} x_{i j}\right) \\
& \sum_{j=1}^{n} Y_{j} \geq 1 \\
& Y_{j} \in\{0,1\} \quad \forall j
\end{aligned}
$$

In the equitation : $\mathrm{m}$ is quantity of demand sort

$\mathrm{n}$ is the alternative partner's quantity

$C_{i j}$ is expenses that i products are offered by partner $\mathrm{j}$. Assume it is only a demand function, generally with the increase which distribute demand here, the expenses are increasing too.

$x_{i j}$ is demand quantity that i product be met from partner $\mathrm{j}$.

$g_{i}$ is fixed cost that select partner $\mathrm{j}$

$Y_{j}$ is 0-1 variables, while choosing partner $\mathrm{j}$, this value is 1 , otherwise 0 .

The decision variable of upper layer programming is $Y_{j}$, because of in the choice at first phase, each strategic partner have already been comprehensive appraised, each supplier basically meet enterprises' requirement from each index, On this basis, we appraise them from price at second phase, because the cooperative partner's selection should be implemented to the price index finally, the goal function make total expense minimum in terms of policymaker. The first restraint guarantee to select one cooperative partner at least, the second restraint is restraint of $0-1$ variable.

The lower-layer programing is to describe the demand distribution among each cooperative partner, obviously, a certain cooperative partner's quantity weight is relevant to its product quality, service level, prestige state, etc . we express it with one quantity weight index $M_{j}, M_{j}$ is the bigger, show that there is more demand that is supplied by cooperative partner $\mathrm{j}$. Enterprise requirement for cooperative partner is different in different time, strategy difference determine quantity weight difference too, for example in one period, enterprises mostly emphasize quality, the company with higher quality will get more quantity weight. However, another factor must be considered ,that is expenses that select $\mathrm{j}$. Generally 
suppose enterprises always consider the factors of two respects at the same time before making decision, attempt to select the partner with the biggest appeal and the least expenses, the mutal function of these two factors will reach the equilibrium between appeal and expenses. Lower layer programing is expressed using mathematics formula:

$$
\begin{array}{lc}
\min T=\sum_{i=1}^{m} \sum_{j=1}^{n} \int_{0}^{x_{i j}} f_{i j}(\omega) d \omega-\sum_{i=1}^{m} \sum_{j=1}^{n} M_{j} x_{i j} \\
\sum_{j=1}^{n} x_{i j}=W_{i} & \\
x_{i j} \leq N Y_{j} & \forall i, j
\end{array}
$$

In the formula: $W_{i}$ express $\mathrm{i}$ products aggregate demand, $f_{i j}$ is inverse function of demand function, $\mathrm{N}$ express positive number arbitrarily. the decision variable of lower layer programing is $x_{i j}$. Goal function express distribution of demand quantity among each cooperative partner and make the total cost minimum . First restraint ensure each demand of product can satisfy, second restraint ensure demand quantity always be distributed among partner selected design, the last restraint is non-negative restraint of the variable.

\section{ALGORITHM OF DOUBLE-LAYER PROGRMING}

if the lower layer programing model of partner selection is considered as a restraint of the upper layer programing in the double layer programing of partner selection. But the existence of the lower layer restraint, this model will be a non- protruding programing, this brings the difficulty for solving the double-layer programing model. This paper propose one kind of method based on genetic algorithm to solve double-layer programing of supply chain partner selection.

The thought of solving double-layer programing based on genetic algorithm is: Confirm the decision variable that is a code scheme of the demand $x_{i j}$, produce the initial colony at random. each individual corresponds to different quantity in the colony. calculate each individual's adaptation degree with the value of goal function, then do genetic calculation to this colony such select, cross and so on. insert each individual into the upper layer and lower layer to solve and get value of goal function, keep the minimum of the sum of two goal functions and its corresponding individual. After many times interaction, algorithm expected to close optimal project, carry on operating described above sequentially, compare the minimum of the goal function of each project, select the optimal project, and then according to individual proportion in the project selected, give the quantity correspondingly to the cooperative partner.

\section{EXAMPLE}

Now set a simple example to prove validity of model and algorithm proposed in this paper.

\section{A Select the strategic partner use grey clustering}

Now a enterprise is evaluating 20 supply chain partners selecting strategic partner (excelletnt) ,alternative supplier(qualified) and non-qualified. the enterprise evaluate those partner in term of four great aspect such as price, service quality, service ability and hardware index. when evaluating, we compare every two partner and score these partner, excellent partner get 1 score, weak partner and equal partner get 0 score. on the basis of this ,apply grey clustering to evaluate those partner ,evaluation type is excellent ,qualified and non-qualified. then select excellent partner as strategic partner. for calculating simpler, we set a example of select 4 supplier.

clustering unit: $\mathrm{i}=1$, partner $1 ; \mathrm{i}=2$,partner 2; $\mathrm{i}=3$,partner $3 ; \mathrm{i}=4$,partner 4 .

(1) Clustering project: $\mathrm{j}=1$,price; $\mathrm{j}=2$,service quality; $\mathrm{j}=3$, service ability, $\mathrm{j}=4$, hardware index.

(2) Evaluation grey clustering: $k=1$,strategic partner, $k=2$, qualified partner; $k=3$,non-qualified partner.

Suppose $d_{i j}$ signify sample which i unit correspond to $\mathrm{j}$ project. evaluation index matrix of four partner $\mathrm{D}$ is:

$$
D=\left[\begin{array}{llll}
d_{11} & d_{12} & d_{13} & d_{14} \\
d_{21} & d_{22} & d_{23} & d_{24} \\
d_{31} & d_{32} & d_{33} & d_{34} \\
d_{41} & d_{42} & d_{43} & d_{44}
\end{array}\right]=\left[\begin{array}{cccc}
15 & 18 & 17 & 18 \\
9 & 0 & 7 & 14 \\
10 & 12 & 10 & 22 \\
3 & 13 & 10 & 7
\end{array}\right]
$$

Determine whitening weight function of NO .j index in NO. k clustering use trigonometric whitening evaluation, appoint it as $f_{k j(x)}$, because of space limitation, we don't list three king grey clustering whitening functions, now we ask clustering weight, the weight which NO .k index correspond to NO. k clustering is:

$$
\omega_{j k}=\lambda_{j k} / \sum_{j=1}^{m} \lambda_{j k}
$$

After calculated we can get:

$$
\begin{aligned}
& \text { when } \mathrm{k}=1 \quad \omega_{11}=0.22 \quad \omega_{21}=0.26 \quad \omega_{31}=0.23 \\
& \omega_{41}=0.29 \\
& \text { when } \mathrm{k}=2 \quad \omega_{12}=0.21 \quad \omega_{22}=0.26 \quad \omega_{32}=0.23 \\
& \omega_{42}=0.30 \\
& \text { when } \mathrm{k}=3 \quad \omega_{13}=0.20 \quad \omega_{23}=0.24 \quad \omega_{33}=0.24
\end{aligned}
$$

$\omega_{43}=0.32$ 
grey coefficient which NO.k partner belong to $\mathrm{k}$

clustering is:

$$
\sigma_{i k}=\sum_{j=1}^{m} f_{j k}\left(d_{i j}\right) \omega_{j k}
$$

After calculated we can get :

$$
\text { when } \mathrm{i}=1 \max _{1 \leq k \leq 3}\left\{\sigma_{1 k}\right\}=\sigma_{11}=0.901 \text {, indicate }
$$

partner 1 belong to strategic partner.

$$
\text { when } \mathrm{i}=2 \max _{1 \leq k \leq 3}\left\{\sigma_{2 k}\right\}=\sigma_{23}=0.486 \text {,indicate }
$$

partner 2 belong to non-qualified partner

$$
\text { when } \mathrm{i}=3 \quad \max _{1 \leq k \leq 3}\left\{\sigma_{3 k}\right\}=\sigma_{32}=0.516 \text {,indicate }
$$

partner 3 belong to qualified partner

$$
\text { when } \mathrm{i}=4 \quad \max _{1 \leq k \leq 3}\left\{\sigma_{4 k}\right\}=\sigma_{43}=0.568
$$

partner 4 belong to non- qulified partner

,indicate

At last, there are 3 partner are excellent, we appoint them as strategic partner and establish a kind of long-term collaboration relationship with them. there are 7 partner is qualified partner, we appoint them as alternative partner, enterprise may help and advise them in preparation for the future under different strategic demands.

\section{B Select optimal partner and its quantity with genetic algorithm}

In the preceding paragraph we have selected three cooperative partner $\left(A_{1}, A_{2}, A_{3}\right)$, now assume there are two kinds demand ( $B_{1}, B_{2}$ ), the aggregate demands of two varieties are $D_{1}=535, D_{2}=762$. Inverse function of the demand function takes the form of the following exponential function:

$$
f_{i j}\left(X_{i j}\right)=a_{j}\left(X_{i j}\right)^{b j}-V_{j} z_{j}
$$

In this $a_{i}, b_{j}$ is parameter, now we suppose

$$
\begin{aligned}
& a_{1}=a_{2}=a_{3}=0.5 \text {, } \\
& b_{1}=b_{2}=b_{3}=0.5 \\
& \text { Suppose } v_{1}=5, v_{2}=3, v_{3}=1 \\
& f_{1}=200, f_{2}=250, f_{3}=300 \\
& M=10, N=1 \\
& g_{1}=1000, g_{2}=900, g_{3}=700 \\
& r_{1}=0.6, r_{2}=0.4, r_{3}=0.5 \\
& s_{11}=s_{12}=s_{21}=s_{22}=1 \\
& t_{1}=0.2, t_{2}=0.3, t_{3}=0.5 \\
& c_{11}=1, c_{12}=3, c_{13}=5, c_{21}=2, c_{22}=4, c_{23}=6
\end{aligned}
$$

The relevant parameters of the genetic algorithm are: individual figure in the colony pop size $=10$, cross probability $p_{m}=0.03$ evolve algebra max Gen Tem=100, adopt the algorithm and Monte Car Lo mould anneal method to calculate double-layer programing respectively, difference was very light finally, it is effective to explain the algorithm. Through Matlab procedure calculation, we can get $z=(1,1,0)$

$X_{11}=298.7632, X_{12}=236.2328, X_{13}=0$

$X_{21}=133.5371, X_{22}=628.4629, X_{23}=0, F=3705.2$,

That is we select partner $A_{1}, A_{2}$,don't select partner $A_{3}$,order quantity from partner 1 is $X_{11}+X_{21}=432.3003<g_{1}=1000$

Order quantity from partner 2 is $X_{12}+X_{22}=864.6957<g_{2}=900$

These can meet ability constraint of cooperative partner.

\section{CONCLUSION}

The cooperative partner's selection is key factor that decide to supply chain management whether succeeds or not, because enterprises must want the cooperative partner to assist to finish in other business when absorbed in one's own key business. So enterprise who adopt supply chain management must treat the cooperative partner's selection question cautiously, find out the appropriate selection method. this paper has set up two-phase cooperative partner selection method on the basis of existing literature. Before setting up supply chain at first, carry on the roughly classification to the existing partner, this paper has used the gray clustering to select the outstanding partner as the strategic cooperative partner, establish long-term cooperation. The second phase is on the basis of the first phase, in each time real application, according to the needs of different strategy, confirm the quantitative index weight, utilize double-layer progrming to build the model. And then utilize genetic algorithm to solve the model, can obtain the quantity that each cooperative partner order. Cite one simple example to demonstrate practicability of method finally.

\section{REFERENCE}

[1]ShihuaMaYongLin.2000.SupplyChain Management [M] Mechanic press,Beijing.4.

[2]Huixia Zou. 2004.Supply Chain Logistics Management. [M].Qinghua University press,8.

[3] Zhou wenkun,Jiang wenchun.supplier selection method based on improved TOPSIS,[J],2005,14(6):39-44 [4]Liu xiao,Li haiyue.the study on the model of supplier selection[J].china science,2004,12(1):139-148 
[5]Dun junong.grey prediction and decision[M].wuhan:huazhong technology press.1986,8 [6]Gao ziyou,Sun huijun.current logistics and transportation system.[M].people transportation.2003. 